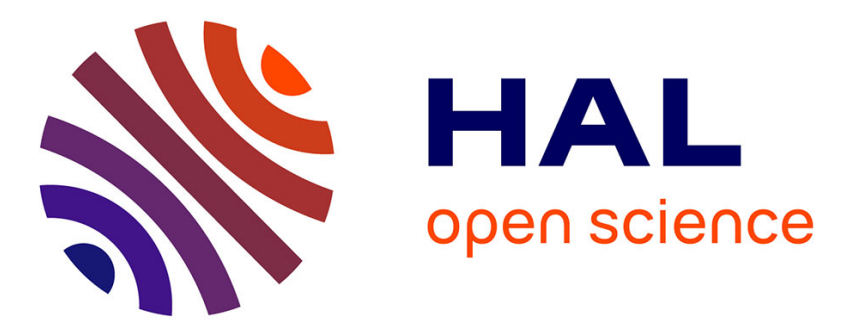

\title{
Cell-DEVS: An Approach to Model the Influence of Social Interactions in Human Behavior
}

\author{
Avneet Behl, Gabriel Wainer, Cristina Ruiz-Martin
}

\section{To cite this version:}

Avneet Behl, Gabriel Wainer, Cristina Ruiz-Martin. Cell-DEVS: An Approach to Model the Influence of Social Interactions in Human Behavior. SummerSim-SCSC, Feb 2018, Bordeaux, France. hal02021837

\section{HAL Id: hal-02021837 \\ https://hal.science/hal-02021837}

Submitted on 16 Feb 2019

HAL is a multi-disciplinary open access archive for the deposit and dissemination of scientific research documents, whether they are published or not. The documents may come from teaching and research institutions in France or abroad, or from public or private research centers.
L'archive ouverte pluridisciplinaire HAL, est destinée au dépôt et à la diffusion de documents scientifiques de niveau recherche, publiés ou non, émanant des établissements d'enseignement et de recherche français ou étrangers, des laboratoires publics ou privés. 


\title{
CELL-DEVS: AN APPROACH TO MODEL THE INFLUENCE OF SOCIAL INTERACTIONS IN HUMAN BEHAVIOR
}

\author{
Avneet Behl \\ Carleton University \\ 1125 Colonel By Dr, K1S 5B6 Ottawa (Canada) \\ abehl@sce.carleton.ca
}

\author{
Gabriel A. Wainer \\ Carleton University \\ 1125 Colonel By Dr, K1S 5B6 Ottawa ON(Canada) \\ gwainer@sce.carleton.ca
}

\author{
Cristina Ruiz-Martin \\ Carleton University and Universidad de Valladolid \\ 1125 Colonel By Dr, K1S 5B6 Ottawa ON(Canada) \\ cruiz@eii.uva.es
}

\begin{abstract}
Human behavior can be influenced by social interactions. The study of how human behavior change has applications in different fields such as marketing, military, psychology, robotics, human-machine interface, anthropology, computational sciences, economics or epidemiology. Applying Modeling and Simulation to study human behavior is encouraged to avoid the long waiting times that takes natural observations of desired events. In our study, we provide a model for human social interaction and influence during a product launch using the Cell-DEVS formalism, the $\mathrm{CD}++$ simulator, Agent-Based Modeling and Bounded Confidence Models. We studied how the size of the market, attributes of an individual and number of people that a human interacts with can affect human behavior.
\end{abstract}

\section{INTRODUCTION}

Social interaction comprises of the actions and reactions of two or more individuals towards one another. It is a common phenomenon; about $32 \%-75 \%$ of the total time that a human walk in a day is spent in simultaneous social interactions (Mehl and Pennebaker 2003). Humans are vulnerable to opinion change under the influence of peers. Modeling of human behavior has applications in marketing, military, psychology, human-machine interface, economics, epidemiology and other fields. Its application in various fields is evolving, as the results of simulation models are able to replicate observed behaviors.

Recent examples of social influence studies include, for instance, a random controlled trial of political messages delivered to 61 million Facebook users during the 2010 US congress elections (Bond et al. 2012); the "chameleon effect", which refers to unconscious mimicry of interacting people (Chartrand and Bargh 1999); a study of changing behavior patterns due to social interactions at the workplace (Chen et al. 2011); a study which consisted of answering a factual question based on aggregated information about the answers of others (Mavrodiev, Tessone, and Schweitzer 2013). While the studies conducted by (Mehl and Pennebaker 2003; Bond et al. 2012; Chen et al. 2011; Mavrodiev, Tessone, and Schweitzer 2013) observed the influence of social interaction on human behavior; (Chartrand and Bargh 1999) claims that individuals even mimic the body postures and movements under social influence. It adds that mimicry increases liking between individuals involved in a social interaction. 
To understand the influence of social interaction on behavior patterns, we need to observe and study humans engaged in a variety of social contexts (Bombari et al. 2015). A major problem in the study of human social interaction and the effect on human behavior using natural observation is the time taken by the desired event to occur naturally in the society with us being present to observe it. For instance, (Baumeister, Vohs, and Funder 2007) discussed the issue of direct observations of behavior being increasingly supplanted by introspective self-reports, hypothetical scenarios, and questionnaire ratings.

In the past, influence patterns have been studied when events take place naturally. In such after-event studies, strong and weak influential factors could be judged, the flow pattern of the propagation could be observed, and the results obtained could be used for the improvement. For example, in the study of Facebook message propagation in (Bond et al. 2012); there was an influence of opinion in the recipients and on their friends, and friends of their friends. The conclusion was that strong ties were more easily influenced than weaker ones. This allowed identifying strong and weak ties as influential factors. Knowing the effect of these factors would be translated on improved planning for similar future events.

Natural observation allows us to study past events. By using this method, it is not possible to study an event before its occurrence (i.e. we do not know what could happen in a future-event). This drawback is mitigated using Modeling and Simulation (M\&S). Using M\&S, we can model individuals in different social situations and simulate how they interact. The emergent behavior of the individuals in these simulated scenarios can be observed and analyzed (Bombari et al. 2015). Using M\&S, we can predict the results of future events based on the data collected from similar previous events, surveys or estimated data. We can use these results to improve the planning of these future events.

Another advantage of using $M \& S$ is that we can observe, study and even predict the effects of social interaction and the influence on human behavior without the long waiting times needed in natural observations. Moreover, we can study scenarios with very low probabilities that are unlikely to happen. Knowing the effects of these improbable scenarios would help us to be prepared for unexpected events.

In this paper, we will present a mechanism for studying the evolution of human opinion due to social interaction and influence using M\&S. We will discuss different models of human social interaction based on the model presented by (Bouanan, Zacharewicz, and Vallespir 2016). Our goal is to provide a serious game, so for example, marketing managers can understand how human social interactions can influence others opinion about a new product launch. We model how the opinion of individuals changes based on the opinion of their neighbors and the degree of influence of each neighbor on the individual. Using this model, we can also understand how different groups of the society (i.e. people with a specific social status or an age range) may influence the opinion of other groups or individuals towards the acquisition of a product or the adoption of a service.

Based on the model presented by (Bouanan, Zacharewicz, and Vallespir 2016), we also use Cell-DEVS formalism, Agent-Based Modeling, Network Diffusion Process, and Bounded Confidence Models (BC Models) to define our model. We identify the agents involved in the communication process, the factors that affect the communication, different scenarios and the actions to be taken in these situations. To implement and simulate the model we use the $\mathrm{CD}++$ simulator (a DEVS and Cell-DEVS simulator).

The model presented by (Bouanan, Zacharewicz, and Vallespir 2016) does not take into account the effects of varying the population size, the predefined threshold to adopt an opinion or the number of neighbors an individual has. To solve these limitations, in this paper, we include these parameters in the model. We perform experiments with two sets of the population with different sizes, we considered two different value of the predefined threshold to adopt an opinion, we study two different neighborhoods and differently generated values of the degree of influence that any individual has upon another individual.

The rest of the paper is organized as follows. In section 2, we present the related work. In section 3, we describe the model and its implementation using the $\mathrm{CD}++$ simulator. In section 4, we present some simulation results. Finally, section 5 states the conclusions of this work and the future research lines. 


\section{RELATED WORK}

As discussed in the introduction, based on the model presented by (Bouanan, Zacharewicz, and Vallespir 2016), we will use Cell-DEVS formalism (based on DEVS), Agent-Based Modeling, Network Diffusion Process, and Bounded Confidence Models (BC Models) to define a model to study the effect of different parameters in the human opinion of a product launch. Our goal is to provide a serious game that managers can use to understand the effects of human social interactions on others opinion.

Discrete Event System Specification (DEVS) (Zeigler, Praehofer, and Kim 2000) is a formalism for modeling complex Discrete Events Systems based on the Set Theory and Systems Theory. The hierarchical and modular structure of DEVS allows defining multiple models that are coupled to work together and modeled by connecting their input and output through messages. In the same way, the resulting model can also be coupled with others models defining multiple layers in the hierarchical structure. In DEVS, atomic models define the behavior of the system, and coupled models describe the structure of the system. The hierarchical and modular structure of DEVS allows model reuse and reduces development and testing times. In DEVS, the model definition, implementation, and simulation are separated. An abstract simulation mechanism is independent of the model itself. This abstract simulator is implemented in different DEVS simulators such as JDEVS (Filippi and Bisgambiglia 2004), DEVSJava (Sarjoughian and Zeigler 1998), CDBoost (Vicino et al. 2015), CD++ (Wainer 2009), etc.

The Cell-DEVS formalism is an extension to DEVS that allows the implementation of cellular models with timing delays (Wainer and Giambiasi 2002, Wainer 2009). A Cell-DEVS model is defined as a lattice of cells in which every cell is a DEVS atomic model, and the cell space is a coupled DEVS model. Using a modular interface, each DEVS basic model can communicate with its neighboring cells in the cell space and with other DEVS models outside the cell space. The neighborhood set can be uniform (i.e., every cell in the space have the same local neighbors) or non-uniform (i.e. each cell can potentially use different neighborhoods). The neighbor cells can be in the local immediacy or they can include remote cells. Each cell has a state, a local transition function and a set of external events that are used to compute the local transition function.

To simulate a Cell-DEVS model, we use the CD++ simulator (Wainer 2009; G. Wainer 2002). This tool simulates both DEVS and Cell-DEVS models. It is an open-source environment built upon Eclipse's extensible mechanisms, which allows adding new plug-ins independently from the rest of the features. It runs either in standalone (single CPU) or in parallel mode (over a network of machines). A dedicated environment called $\mathrm{CD}++$ Builder has been created to ease M\&S. CD++ Builder is a Graphical User Interface (GUI) for the $\mathrm{CD}++$ simulator built upon Eclipse's extensible mechanisms. $\mathrm{CD}++$ supports different languages for specifying DEVS models reducing the barrier entrance for non-developer users. While the architecture and the implementation of $\mathrm{CD}++$ simulator allow simple definition and reuse of components, the extended architecture supports features allowing the cells to use multiple state variables as required by our model; without adding much complexity.

In $\mathrm{CD}++$, the local transition function is defined as a set of rules. They are implemented following the $\mathrm{CD}++$ high-level language with the form:

\section{rule: POSTCONDITION DELAY \{ PRECONDITION \}}

This language indicates that when the PRECONDITION is satisfied, the state of the cell will change to the designated POSTCONDITION, whose computed value will be transmitted to other components after consuming the DELAY. If the precondition is false, the next rule in the list is evaluated until a rule is satisfied or there are no more rules.

Agent-Based Modeling (ABM) is a method in which a system is modeled as a set of autonomous decision-making entities called agents. Each agent studies the situation independently. A system may have one or multiple agents. The agent is a physical or virtual entity (it is a human in the model developed in this work), that interacts with the environment and is able to make decisions in order to meet its 
objectives (Jennings, Sycara, and Wooldridge 1998). The simplest Agent-Based Model is a set of agents and a set of relationships between them. Complex Agent-Based Models can use neural networks or machine learning algorithms to imitate the learning process that takes place in the real world (Bonabeau 2002). The main advantage of ABM is the direct correlation between agents and the entities in the real system. There is also a direct correlation between the interactions in the model and the relations in the real world (Edmonds 2001). One of the disadvantages of ABM is the lack of a mathematical representation of the model (Galán et al. 2009). This lack of formal representation has advantages and disadvantages. Although we can introduce errors in the code and artifacts when implementing the model, it is easy to observe the emergent behavior of the system.

In this work, the Agent-Based Model will define a set of relations between the entities (i.e. a network) and a diffusion process over this network. A diffusion process is cascading process that allows us to understand the effects of different attributes on the spread of different elements. For example, it can be the spread of an opinion based on age or the spread of virus based on temperature and humidity.

When simulating diffusion process of human behavior adoption, the agents (that form a social network) may be divided into clusters based on their behavior. At a certain time, these clusters may lead to polarization. When the network is polarized (i.e. divided into poles of agents with extreme behaviors), the diffusion process of human behavior ceases to occur because all agents will have neighbors with a behavior either too similar or too different to be influenced. The combination of ABM and Bounded Confidence Models (BC Models) can prevent this polarization.

An early formulation of BC Model was given by (Deffuant et al. 2000). To avoid polarization, BC Model prevents creating extreme agents by only allowing the interaction of those agents whose opinions are close enough (i.e. within a bounded interval). If we use BC Models, the opinion is continuous. The advantage of using continuous opinions is that we can measure the closeness of the opinions between two agents more easily than if they were discrete. This makes a gradual influence on the opinion of the neighbors and prevents the polarization (Lorenz 2007). To check the closeness of opinion, a tolerance threshold is defined. The agents with an opinion difference below the threshold may interact and influence each other; the agents with an opinion different above the threshold will have no influence in each other (Hegselmann and Krause 2002; Lorenz 2007). The number of agents in an opinion cluster increases in steps when the tolerance threshold is decreased (Weisbuch 2004). Therefore, BC Models avoid the polarization by not generating the extremes.

(Bouanan, Zacharewicz, and Vallespir 2016) used this combination of ABM and BC Models to study human behavior. They model the evolution of agent's opinion using Cell-DEVS formalism to validate the use of DEVS and Cell-DEVS in the study social influence using ABM. They concluded that at the atomic level, DEVS models integrate the set of attributes (i.e. static attributes such as gender, age, and languages), the variables (i.e. dynamic attributes such as opinion, satisfaction degrees, and interests) and the behavioral functions associated with an agent. These atomic models were coupled to form a CellDEVS network. They highlighted that the decoupling of the model and the simulator (they used the CD++ Builder to implement their model) has enabled the modeler to focus on modeling the behavior of the agents. Through their simulation, they obtained a better understanding of how individual behavior and information can affect the whole population.

The focus of the authors was to demonstrate the applicability of DEVS and Cell-DEVS to study the influence of social interactions in human behavior. Therefore, their model does not consider the effects of changing the size of the population, the predefined threshold for opinion adoption or the neighborhood set. They choose ten randomly individuals at the initial stage to study the influence in the whole population set. To solve these limitations, we extend (Bouanan, Zacharewicz, and Vallespir 2016) work by modeling the influence of social interactions on human behavior to provide a serious game for managers in order to understand the effect of different parameters in the spread of an opinion. We provide a model to observe the effect of changing the population size, the threshold value of adopting an opinion and the neighborhood set. To conduct the experiments, we consider two sizes for our model; one having 
400 agents and another having 1600 agents. We use both Moore and Von Neumann neighborhood although more complex neighborhoods (such as not uniform neighborhood with remote cells) can be studied using our model. Likewise, we experiment with different values for the opinion adoption threshold. We also observe how influence patterns change due to random and calculated values for the degree of influence of one agent in the others (which is an attribute of the agent).

\section{MODEL DESCRIPTION}

We modeled the evolution of human behavior under social influence using the Cell-DEVS formalism, ABM, Network Diffusion Processes and BC Models. We implement the model using the CD++ simulator. We extend the model presented in (Bouanan, Zacharewicz, and Vallespir 2016) in order to study the effects of size of the population (i.e. number of individuals), the number of people that an individual interacts with (i.e. the neighborhood size), the threshold value of adopting an opinion or behavior, and the values for the degree of influence of one agent in the others.

Using ABM, we model humans as agents (i.e. independent entities with their own attributes and behavior rules). We represent each agent as a set of static attributes: (1) age group, (2) degree of influence and (3) social class. Each agent also has a set of dynamic attributes that evolve as the agents interact: (1) opinion and (2) state. We can vary the static attributes to simulate different scenarios.

Using Cell-DEVS, we represent each agent as a 2-D cell. The formalism further couples these cells to imitate interactions within individuals. Different neighborhood sets can be used to couple the cells. We can study the effects of different social connections varying the neighborhood. The attributes of the agents (both dynamic and static) are represented by different state variables on each cell.

The dynamic attribute "state" can take three possible values: informed (A), uninformed (B) and passive (C) as shown in figure 1. Based on the current state, each agent decides the action to be taken.

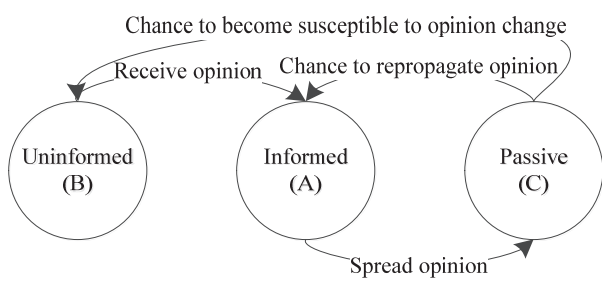

Figure 1: Transitions among the three states of an agent.

Uninformed agents (state B) are open to be influenced. An individual can be influenced from another individual in the neighborhood if the difference in their opinion falls below a predefined threshold value (a parameter of the model); an agent is simultaneously affected by all the neighbors who satisfy the threshold condition. The resulting opinion depends on the degree of influence associated with the neighbors. Once influenced, the state changes to informed (state A). An informed agent (state A) is ready to influence their neighbors, but cannot be influenced. After it spreads its opinion to the neighbors, its state changes to passive (state C). A passive agent (state C) cannot be influenced by a neighbor or influence other neighbors. Passive agents will be given a chance to re-propagate their opinion (state A) or become susceptible to opinion change (state B) after a delay. We reactivate agents because, in real life, humans spread their opinion more than once if they feel that they can influence other people and there are susceptible to opinion change multiple times. Moreover, we avoid a dead situation where all agents are passive and there is no more flow of messages in the population set.

As shown in the state diagram of figure 1, we can see that only an uninformed agent can be influenced by opinions and become informed; only an informed agent can spread its opinion and become passive. Every passive agent gets a chance to become informed or uninformed again. 
The initial values of the opinion (dynamic attribute) of the agents are generated using a predefined function. They are on the scale from 0 to 10 . Each opinion value is associated with a color to observe how the opinion (i.e. behavior) of the agents evolves. The opinion is updated when the agent evolves from uninformed to informed.

We define the behavior of the agents using the following set of rules: (1) the initialization rule, (2) the informed state rule, (3) the uninformed state rule and (4) the passive state rule. The following rules show how the evolution of human opinion evolves due to social interactions.

The initialization rule (figure 2) is used to initialize the cell space. This rule generates random initial values for opinion, age group, class, and state and initializes them. As we have already mentioned the state can be informed, uninformed or passive. The initialization rule is triggered when we are in the initial state (i.e., \$initialFrame is 1). These values are transmitted as outputs through the corresponding ports after the delay (i.e. immediately). The value stored in the variable "class" is assigned to the output of the port "class". This value is generated using the function "\$class: $=1+\operatorname{randInt}(2)$ ". Using this function, we randomly generate integers within the range of 1 and 3 and assign them to the state variable "class" of the cells. Each value (i.e. 1, 2, and 3) represents a social class. Similarly, we randomly generated the values for the variables "age group", "state" and "opinion". The values of these variables are assigned to the ports with the same name. Although in figure 2 we calculate the degree of influence (i.e. "influence") based on the age group and class (i.e. "\$influence:=\$agegroup * \$class"), this value can also be randomly generated. In section 4, we study the effect of having random influence or pre-calculated influence.

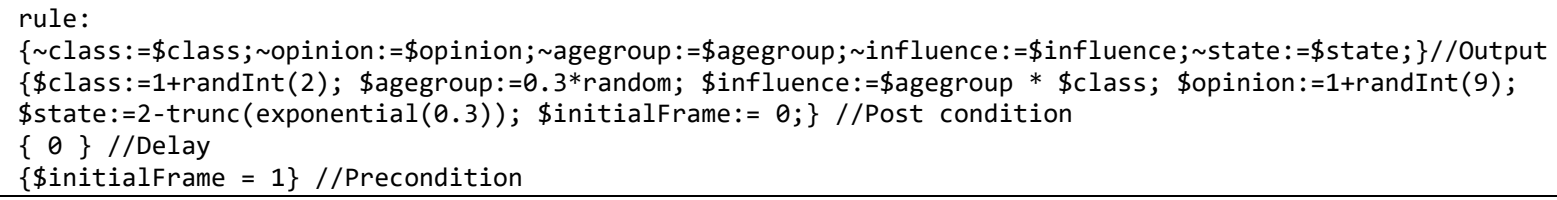

Figure 2: Initialization rule for the agents implemented in $\mathrm{CD}++$.

The informed state rule (figure 3 ) is used to compute the next state for informed agents (i.e. state A). In the precondition of the informed state rule, we check if an agent is in the state 'informed' (computed by macro (A)). If this condition is met, the agent changes its state to "passive" (computed by macro(C) ). The opinion remains the same. After a time delay of $100 \mathrm{~ms}$, we transmit the outputs (i.e. opinion and state). This means that although an agent in 'informed' state influences their neighbors, it cannot be influenced.

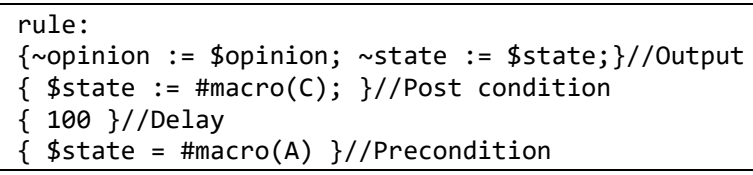

Figure 3: Informed state rule for the agents implemented in $\mathrm{CD}++$.

The rule that governs the influence of an uninformed agent (i.e. state B) is shown in figure 4.

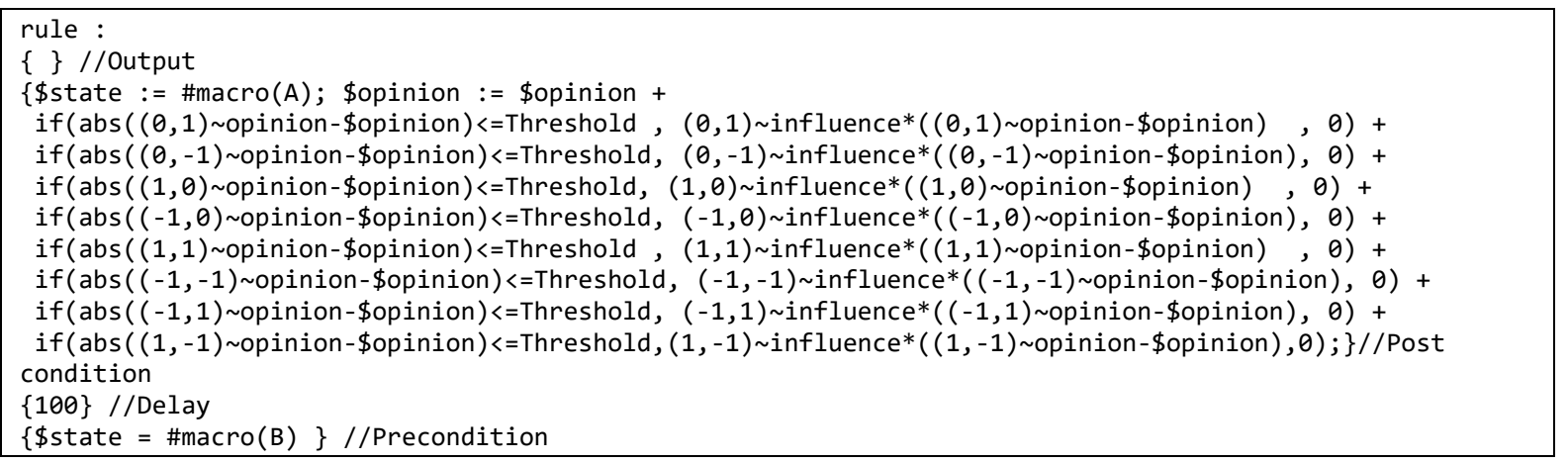

Figure 4: Uninformed state rule for the agents implemented in $\mathrm{CD}++$ (Moore neighborhood). 
If the state of the agent is "uninformed" (computed by macro (B) in the precondition), both the opinion and the state variables are updated. The agent changes its state to 'informed" (computed by macro(A)). The opinion is update following equation (1).

$$
O(x)=O(x)+\sum_{\begin{array}{c}
y \in \text { neighborhood } \mid \\
|O(x)-O(y)| \leq \text { Threshold }
\end{array}} \text { influence } *[O(y)-O(x)]
$$

Where:

$x$ is the agent to be influenced

$O(x)$ is the current opinion of an agent to be influenced

$O(y)$ is the current opinion of an agent in the neighborhood of $x$

influence is the degree of influence of agent $y$ over agent $x$ (it is an attribute of the agent as we have already explained)

Threshold is a parameter of the model that determines the bounded interval. It is the threshold between the differences of two opinions in order to consider agent $y$ in the set of agents that influence agent $x$.

Equation (1) is implemented in the rule presented in figure 4 using the $\mathrm{CD}++$ simulator notation. It is implemented using the Moore neighborhood. The equation is implemented similarly for other neighborhood types. As we can see in figure 4, the agent does not generate any output. This rule means that an uninformed agent is susceptible to be influenced but do not influence other agents.

The passive state rule (figure 5) is used to compute the next state for passive agents (i.e. state C). Using this rule, the state of passive agents (checked using macro (C)) is updated to informed, uninformed or passive giving them the chance to spread their opinion again or to be susceptible to be influenced after a random delay. A random disturbance is also included in the current opinion of the agent.

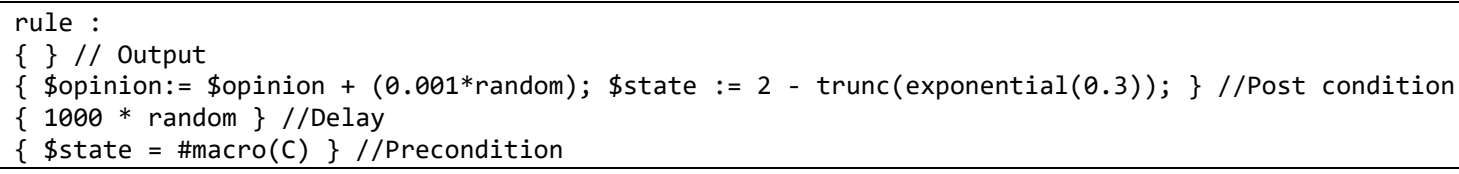

Figure 5: Passive state rule for the agents implemented in $\mathrm{CD}++$.

We use this model to observe the change in human behavior patterns induced by the change in population size, neighborhood size, the threshold value and degree of influence of neighbor.

\section{SIMULATION RESULTS}

In this section, we discuss different scenarios showing the simulation results of social influence on human behavior under varying conditions. We consider:

- Changes in the population size: two sets of the population, 1600 and 400 agents represented by 40x40 and 20x20 2D-cell square grids respectively

- Changes in the threshold value: two threshold values: 2 and 3

- Changes in the neighborhood: two neighborhood types: Moore and Von Neumann neighborhood

- Changes in the degree of influence: two ways to calculate the degree of influence: randomly and as a function of age group and class

Using this study we observe the effect of considering a BC Model. According to the BC model, the behavior patterns will not reach the polarization stage if only the mutually oriented neighbors interact (i.e. only individuals whose difference of opinion is below a certain threshold level interact). We also see how increasing the neighborhood in the model helps us to consider more individuals that can influence the opinion of another individual. In addition, different sizes of the neighborhood can represent different 
levels of connectivity among people. For example, a larger neighborhood is analogous to a population set which is more social; a smaller neighborhood is analogous to a population set which is less social.

A major factor in the evolution of human behavior (or opinion) is the degree of influence of their neighbors, which defines how much an individual can influence a neighbor. The neighbor with the higher degree of influence will have a greater impact on an individual's opinion than a neighbor with the lower degree of opinion. We initially studied the influence of an individual on another by using a random degree of influence. Choosing random values of the degree of influence is not realistic and it defeats the purpose of M\&S. To have a better estimate how opinion will be evolved we should calculate the degree of influence rather than randomly generate it. In this first approach to the model, we calculate the degree of influence based on the social class and age group. More accurate formulas constructed from observations from previously conducted surveys or studies can also be incorporated in the model.

In the rest of the section, we use different colors to represent each sub-range of the opinion of the agents. Yellow cells represent agents with opinion between 0 and 3, orange cells represent agents with opinion between 3 and 6 and red cells agents with opinion between 6 and 10. We want to remark that the cells do not represent the physical position of the actual individuals, but their relationship with the individuals they influence or are influenced by.

The first scenario (figure 6) consists of a model with 1600 agents, a threshold value for opinion adoption of 2, Von Neumann neighborhood and randomly generated initial values for the individual's degree of influence. As the simulation evolves, we observe how the opinions of individuals change due to social interactions represented by changing the colors (compare the right and the left frames of figure 6). Clusters of individuals with homogeneous opinions begin to form (see orange and red clusters in the central and left frame of figure 6). As the simulation evolves, the size of the clusters increases although there is no polarization thanks to the BC Model.

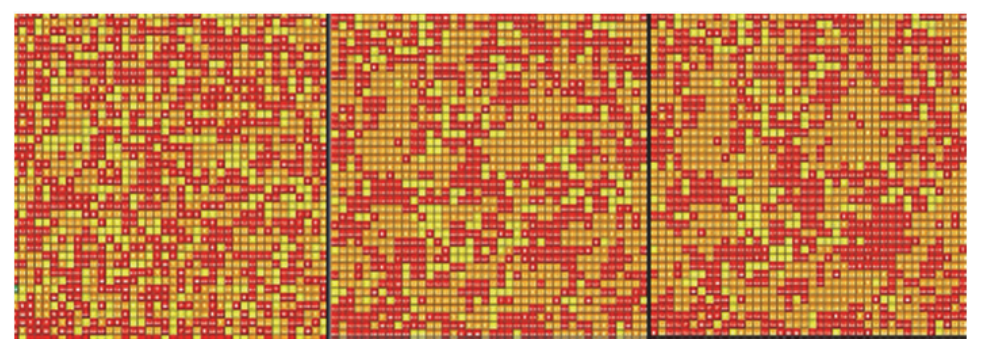

Figure 6: 1600 individuals, threshold=2, Von Neumann Neighborhood, random degree of influence.

In a second scenario (figure 7), we replace the randomly generated degree of influence by a new calculated version based on the social class and age group as discussed in Section 3. This calculated degree of influence introduces two new attributes associated with an individual; age group and class. Comparing the simulation results of figure 6 and 7, we observe a change in the evolution of the behavior patterns. Having a calculated degree of influence is more realistic than having a random one. Therefore, introducing more relevant attributes in the behavior of an individual will lead to more accurate results.

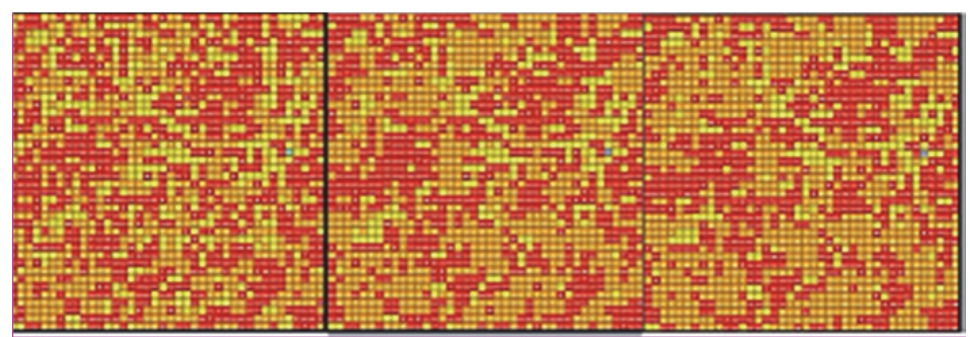

Figure 7: 1600 individuals, threshold=2, Von Neumann Neighborhood, calculated degree of influence. 
In a third scenario (figure 8), we further modified our model extending the neighborhood from Von Neumann to Moore. Increasing the neighborhood size, we observe (comparing the left frames of figure 6, 7 and 8) that we get a more homogeneous behavior. Most of the agents have an opinion in the range 3-6 (orange). Moreover, we noticed that the number of individuals with the least dominant opinion (i.e. range 0-3 - yellow) decreases. Comparing the left frame of the three scenarios (figures 6, 7 and 8) we observe that the clusters are bigger and there is no polarization in the opinion of the agents. Using the BC Model, we observe that the larger group of agents at the end of the simulation is formed by those ones with a moderate opinion (i.e. range 3-6 - orange).

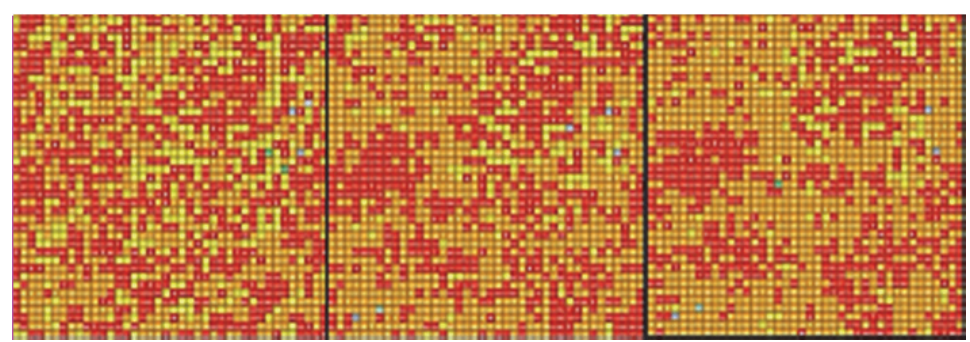

Figure 8: 1600 individuals, threshold=2, Moore Neighborhood, calculated degree of influence.

In a fourth scenario (figure 9), we reduce the population size to 400 individuals keeping the other parameters as in scenario 3. We can observe (comparing the actual scenario with the ones previously discussed) that we get a more homogeneous behavior as we reduce the population size (see that in figure 9 left frame, most agents fall in the 3-6 opinion range - orange).

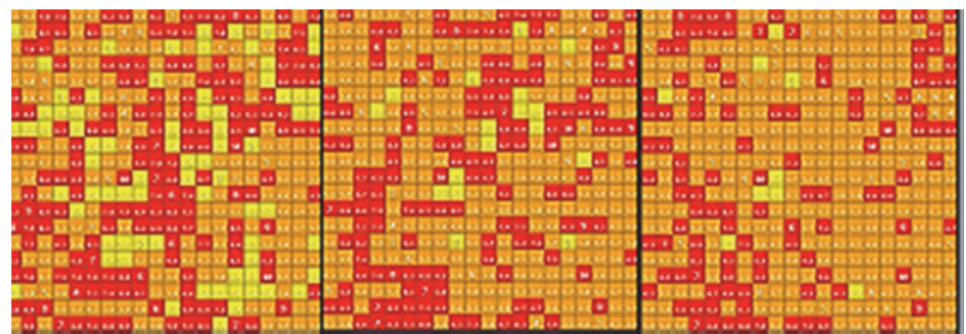

Figure 9: 400 individuals, threshold=2, Moore Neighborhood, calculated degree of influence.

In a fifth scenario (figure 10), we test the increase of the threshold value for opinion adoption from 2 to 3 . Comparing the simulation results in figure 9 and 10, we observe that the number of individuals in a cluster of homogeneous opinions decreases when we increase the threshold value. Agents are more susceptible to opinion change even if the opinion is far away. These results suggest that the accuracy of the BC Model has a great influence on the accuracy of the simulation results.

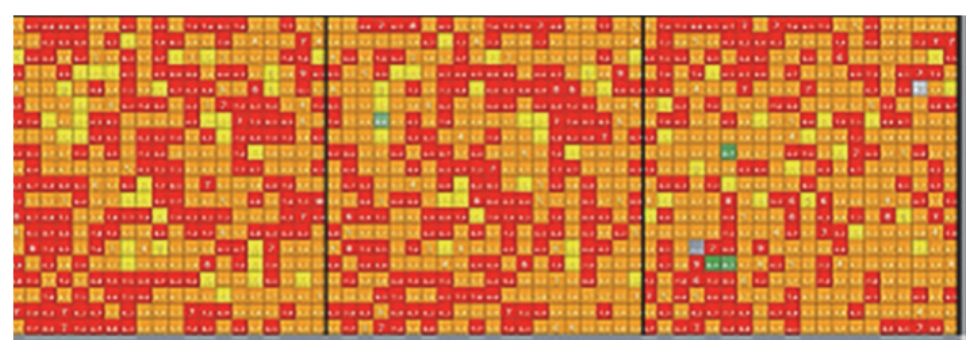

Figure 10: 400 individuals, threshold=3, Moore Neighborhood, calculated degree of influence.

In a sixth scenario (figure 11), we observe that the density of clusters is further reduced when we reduce the neighborhood size (to Von Neumann) and the threshold value (from 3 to 2). 


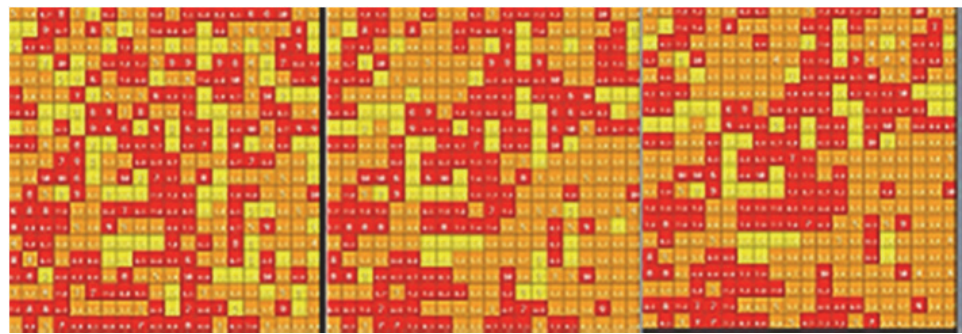

Figure 11: 400 individuals, threshold=2, Von Neumann Neighborhood, calculated degree of influence.

In the next scenario (figure 12), we increase the threshold value from 2 to 3 keeping the rest of the parameters as in the sixth scenario. In this case, we observe an increase in the density of the clusters. However, in scenario five (figure 10), we observe that the number of individuals in a cluster of homogeneous opinions decreases with an increase in the threshold value. This suggests a combined effect of the neighborhood and the threshold value. As we already mentioned we first need to find an accurate BC Model, and then, study the effect of different neighborhoods in the human behavior adoption.

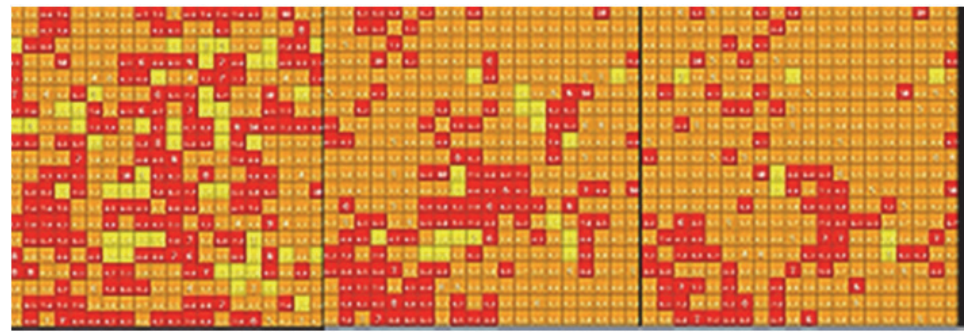

Figure 12: 400 individuals, threshold=3, Von Neumann Neighborhood, calculated degree of influence.

Finally, we consider a change in the way the degree of influence is calculated (figure 13). The rest of the parameters are kept as in the previous scenario. In figure 13, we observed a considerable reduction in the density of clusters if we compare with the results in figure 12. As already observed in figure 6 , we observe again in this scenario that the results really differ from the ones in figure 12 . The results in this scenario confirm that an accurate degree of influence is important to obtain accurate results from the model.

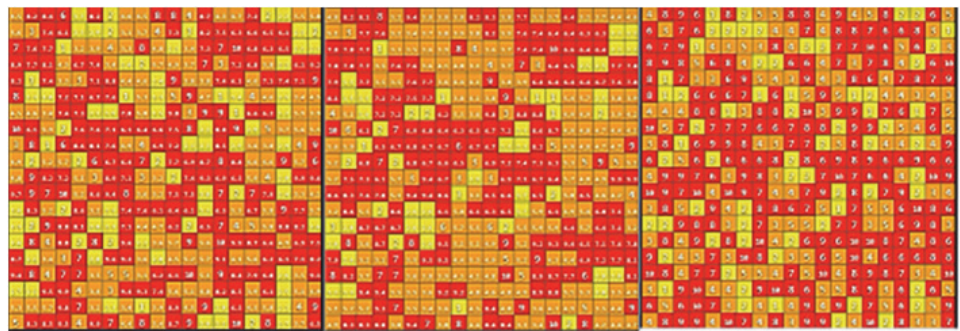

Figure 13: 400 individuals, threshold=3, Von Neumann Neighborhood, random degree of influence.

\section{CONCLUSIONS}

Human behavior is susceptible to social influences. The study of how human behavior evolves has numerous applications in different fields such as psychology, marketing, robotics, etc. Applying M\&S to study human behavior is encouraged since it solves some of the drawbacks of natural observation (e.g. long waiting observation times, not being able to observe low probability situations, etc.) In this work, we modeled human behavior to observe changes in human behavior patterns due to social influence. We use the Cell-DEVS formalism and ABM. We introduced also a BC model to avoid polarization in the opinion of the agents. The simulations were performed using the $\mathrm{CD}++$ simulator. We studied changes in human 
behavior patterns induced by changes in the population size, the neighborhood size, the threshold value for opinion adoption, and the degree of influence of the agent.

Though our study, we observe that the results were more similar to real situations when we replace the randomly generated value of the degree of influence by calculated values. They were more close to real scenarios when we extended the size of the neighborhood. By varying size of the population and changing the threshold values of opinion adoption, we observed that the density of clusters with individuals having homogeneous ideas changed. Although the density of clusters increases in some cases, a stage of polarization was not achieved thanks to the application of a BC Model.

We could easily make changes in the population size, the neighborhood size, the threshold value and the value of the degree of influence using the Cell-DEVS formalism and the CD++ simulator. Having an agent on each cell allowed the agents to act independently based on their attributes (both static and dynamic). This enabled us to observe the impact of changing attributes and their values on behavior patterns. Observing how this attributes influence the behavior of people is useful for example, for marketing managers when they plan to launch a product. With this model, we aim to provide them a sample model that they can customize and calibrate modifying the attributes of the agents and their behavior about opinion adoption to adapt it to their specific case studies. Managers can use this model as a serious game to understand the impact of different parameters in the human opinion and behavior adoption.

Future research will aim to add more attributes that can influence the behavior of the agents. We will also analyze more realistic neighborhoods that may affect an individual such as non-uniform neighborhoods that include remote cells.

\section{REFERENCES}

Baumeister, Roy F., Kathleen D. Vohs, and David C. Funder. 2007. "Psychology as the Science of SelfReports and Finger Movements: Whatever Happened to Actual Behavior?" Perspectives on Psychological Science 2 (4): 396-403. doi:10.1111/j.1745-6916.2007.00051.x.

Bombari, Dario, Marianne Schmid Mast, Elena Canadas, and Manuel Bachmann. 2015. "Studying Social Interactions through Immersive Virtual Environment Technology: Virtues, Pitfalls, and Future Challenges." Frontiers in Psychology 6 (June). doi:10.3389/fpsyg.2015.00869.

Bonabeau, E. 2002. "Agent-Based Modeling: Methods and Techniques for Simulating Human Systems." Proceedings of the National Academy of Sciences 99 (Supplement 3): 7280-87.

Bond, Robert M., Christopher J. Fariss, Jason J. Jones, Adam D. I. Kramer, Cameron Marlow, Jaime E. Settle, and James H. Fowler. 2012. "A 61-Million-Person Experiment in Social Influence and Political Mobilization.” Nature 489 (7415): 295-98. doi:10.1038/nature11421.

Bouanan, Youssef, Gregory Zacharewicz, and Bruno Vallespir. 2016. "DEVS Modelling and Simulation of Human Social Interaction and Influence." Engineering Applications of Artificial Intelligence 50 (April): 83-92. doi:10.1016/j.engappai.2016.01.002.

Chartrand, Tanya L., and John A. Bargh. 1999. "The Chameleon Effect: The Perception-behavior Link and Social Interaction." Journal of Personality and Social Psychology 76 (6): 893-910.

Chen, Chih-Wei, Asier Aztiria, Somaya Ben Allouch, and Hamid Aghajan. 2011. "Understanding the Influence of Social Interactions on Individual's Behavior Pattern in a Work Environment." In Human Behavior Understanding, 146-57. doi:10.1007/978-3-642-25446-8_16.

Deffuant, Guillaume, David Neau, Frederic Amblard, and Gérard Weisbuch. 2000. "Mixing Beliefs among Interacting Agents." Advances in Complex Systems 3 (01n04): 87-98.

Edmonds, Bruce. 2001. "The Use of Models - Making MABS More Informative An Analysis of Modelling Understanding Multi-Actor Systems Through Modelling With MAS Abstraction 
Analysis of Target System."

Filippi, Jean-Baptiste, and Paul Bisgambiglia. 2004. "JDEVS: An Implementation of a DEVS Based Formal Framework for Environmental Modelling." Environmental Modelling \& Software 19 (3): 261-74. doi:10.1016/j.envsoft.2003.08.016.

Galán, José Manuel, Luis Izquierdo, Segismundo Izquierdo, José Ignacio Santos, Ricardo del Olmo, Adolfo Lopez-Paredes, and Bruce Edmonds. 2009. "Errors and Artefacts in Agent-Based Modelling." Journal of Artificial Societies and Social Simulation 12 (1).

Hegselmann, Rainer, and Ulrich Krause. 2002. "Opinion Dynamics and Bounded Confidence Models, Analysis, and Simulation." Journal of Artifical Societies and Social Simulation (JASSS 5 (3).

Jennings, N. R., K. Sycara, and M. Wooldridge. 1998. "A Roadmap of Agent Research and Development." Autonomous Agents and Multi-Agent Systems 38: 7-38.

Lorenz, Jan. 2007. "Continuos Opnion Dynamics under Bounded Confidence: A Survey." International Journal of Modern Physics C 18 (12): 1819-38. doi:10.1142/S0129183107011789.

Mavrodiev, Pavlin, Claudio J. Tessone, and Frank Schweitzer. 2013. "Quantifying the Effects of Social Influence." Scientific Reports 3 (1): 1360. doi:10.1038/srep01360.

Mehl, Matthias R., and James W. Pennebaker. 2003. "The Sounds of Social Life: A Psychometric Analysis of Students' Daily Social Environments and Natural Conversations." Journal of Personality and Social Psychology 84 (4): 857-70. doi:10.1037/0022-3514.84.4.857.

Sarjoughian, Hessam S, and Bernard P Zeigler. 1998. "DEVSJAVA: Basis for a DEVS-Based Collaborative M \& S Environment." In Proceedings of SCS International Conference on Web-Based Modeling and Simulation. San Diego.

Vicino, Damian, Daniella Niyonkuru, Gabriel Wainer, and Olivier Dalle. 2015. "Sequential PDEVS Architecture." In DEVS '15 Proceedings of the Symp on Theory of M\&S: DEVS Integrative $M \& S$ Symposium, 165-72.

Wainer, Gabriel A.. 2009. "Discrete-Event Simulation: a practitioner's approach" CRC Press. 2009.

Wainer, Gabriel A., and Norbert Giambiasi. 2001. "Application of the Cell-DEVS Paradigm for Cell Spaces Modelling and Simulation." SIMULATION 76 (1): 22-39.

Wainer, Gabriel A, and Norbert Giambiasi. 2002. "N-Dimensional Cell-DEVS Models." Discrete Event Dynamic Systems 12 (2): 135-57. doi:10.1023/A:1014536803451.

Weisbuch, G. 2004. "Bounded Confidence and Social Networks." The European Physical Journal B Condensed Matter 38 (2): 339-43. doi:10.1140/epjb/e2004-00126-9.

Zeigler, Bernard P, Herbert Praehofer, and Tag Gon Kim. 2000. Theory of Modeling and Simulation: Integrating Discrete Event and Continuous Complex Dynamic Systems. Academic press.

\section{AUTHOR BIOGRAPHIES}

AVNEET BEHL obtained a Meng graduate in Systems and Computer Engineering (Carleton University). She received a Bachelor of Technology degree in Electronics and Communication Systems from Guru Nanak Dev University. Her email address is avneet.behl@alumni.carleton.ca.

GABRIEL WAINER is Professor at the Department of Systems and Computer Engineering at Carleton University. He is a Fellow of the Society for Modeling and Simulation International (SCS). His email address is gwainer@sce.carleton.ca.

CRISTINA RUIZ-MARTIN has obtained a Ph.D. in Industrial Engineering (University of Valladolid, UVa) and Systems and Computer Engineering (Carleton University). She received an Engineering degree, a M.Sc. and a Master in Project Management from UVa. Her email address is cruiz@eii.uva.es 